\title{
A fast construction method for VLSI power and ground pin assignment
}

\author{
Shundao Xie ${ }^{1,2, a}$, Yaogan Liang ${ }^{1,2, b}$, Rongjun Chen ${ }^{3, c}$, Mushui Zhang ${ }^{1, d}$ and \\ Hongzhou Tan ${ }^{1,2}$, e \\ ${ }^{1}$ School of Electronics and Information Technology, Sun Yat-Sen University, Guangzhou, China \\ ${ }^{2}$ SYSU-CMU Shunde International Joint Research Institute, Foshan, China \\ ${ }^{3}$ Guangdong Polytechnic Normal University, Guangzhou, China

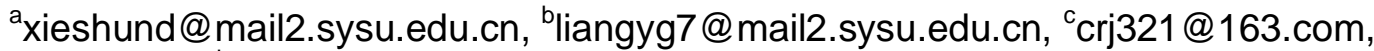

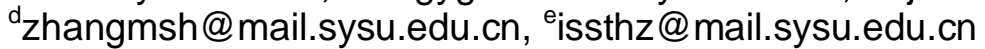

Keywords: signal integrity, pin assignment, prior knowledge, construction method

\begin{abstract}
In this paper, a fast pin assignment optimization method is proposed for improving signal integrity performance of VLSI package. This performance is evaluated by two cost function. Some other methods based on heuristic algorithm can find a good solution for pin assignment problem. Since the solution space is of high dimension, those algorithms require a large time consumption. The proposed pin assignment construction method is guided by the prior knowledge about the optimal solution of pin assignment. It generates a solution by directed construction. Experimental results show that the quality of solution produced by proposed method is better than that by heuristic algorithm and the time consumption is practically negligible.
\end{abstract}

\section{Introduction}

With the rapid increase in VLSI performance and the associated enhancement in their functions, more and more functional circuits are integrated into a single chip. Signal integrity problem due to power and ground $(\mathrm{P} / \mathrm{G})$ pin arraignment turns into a critical problem in VLSI designing[1].

Since the pin assignment problem is NP-complete [2] which conventional optimizer cannot solve. There has been some work in pin assignment problem with heuristic algorithm being implemented. In [3], the inductance matrix $\boldsymbol{L}$ of the package are extracted to calculate the ground noise voltage, then the optimization problem is to minimize it. Recently, [4] has proposed two cost function models to evaluate the mutual inductance and return path quality. They are combined into a single fitness function and the problem is also to minimize it. Heuristic algorithms such as genetic algorithm (GA) and simulate anneal (SA) are used in [4][5]. These algorithm works well but consumes too much time for iterative computation. However, the optimal solutions are regular. With this prior knowledge, it's possible to find a relatively good solution in an extraordinarily short time. This paper focus on reducing time consumption when dealing with large-scale packages.

\section{Preliminary}

For a given package (Fig. 1 (a)), all the P/G pin are numbered. A distance matrix $\boldsymbol{D}$ (Fig. 1 (b)) is derived where its element $d_{i j}$ is the distance between pin $\mathrm{i}$ and pin $\mathrm{j}$.

If the number of $\mathrm{P} / \mathrm{G}$ pin is $n, \boldsymbol{D}$ is of $n \times n$ dimension. The elements on its diagonal are replaced with $d_{\max }$, which is larger than any element in $\boldsymbol{D}$ and insure the value of the logarithm is positive. To evaluate the power integrity (PI) and signal integrity (SI) performance of a package, two cost functions are derived [4]. 


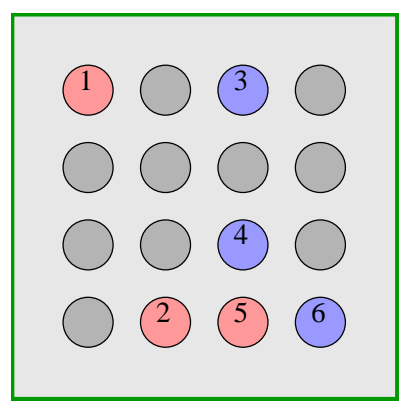

(a) $4 * 4$ Package

$$
\boldsymbol{D}=\left[\begin{array}{cccccc}
d_{\max } & 3.16 & 2 & 2.83 & 3.61 & 4.24 \\
3.16 & d_{\max } & 3.16 & 1.41 & 1 & 1 \\
2 & 3.16 & d_{\max } & 2 & 3 & 3.16 \\
2.83 & 1.41 & 2 & d_{\max } & 1 & 1.41 \\
3.61 & 1 & 3 & 1 & d_{\max } & 1 \\
4.24 & 2 & 3.16 & 1.41 & 1 & d_{\max }
\end{array}\right]
$$

(b) Distance Matrix

Here $M_{\text {sum }}$ is derived for the PI:

Fig. 1 Construction of Distance Matrix

where

$$
M_{\mathrm{sum}}=\sum_{i=1}^{N} \sum_{j=1}^{N} a_{i j} \ln \left(\frac{d_{\mathrm{max}}}{d_{i j}}\right) \#(1)
$$

And $D_{\text {sum }}$ is for SI:

$$
a_{i j}=\left\{\begin{array}{l}
-1, \text { when current between pin } \mathrm{i} \text { and pin } \mathrm{j} \text { are in opposite direction } \\
0, \text { when } \mathrm{i}=\mathrm{j} \\
1, \text { when current between pin } \mathrm{i} \text { and pin } \mathrm{j} \text { are in same direction }
\end{array}\right.
$$

$$
D_{\text {sum }}=\operatorname{var}\left(\boldsymbol{d}_{\text {min }}\right)-\frac{1}{N} \sum_{j=1}^{N} q_{j} \#(2)
$$

where var denotes to get variance, $q_{j}$ is the smallest element of the jth column of $\boldsymbol{D}$ and $\boldsymbol{d}_{\min }$ is a vector:

$$
\boldsymbol{d}_{\min }=\left[\begin{array}{llll}
q_{1} & q_{2} & \cdots & q_{N}
\end{array}\right] \#(3)
$$

Because $M_{\text {sum }}$ vary in a relatively large range and $D_{\text {sum }}$ in a small one. $D_{\text {sum }}$ is multiplied by a relatively large weight $w$. Fitness function $f$ for single object genetic algorithm is equal to the summation of $M_{\text {sum }}$ and weighted $D_{\text {sum. }}$. In general, a better result has a smaller $f$ value.

$$
f=M_{\text {sum }}+w D_{\text {sum }} \#(4)
$$

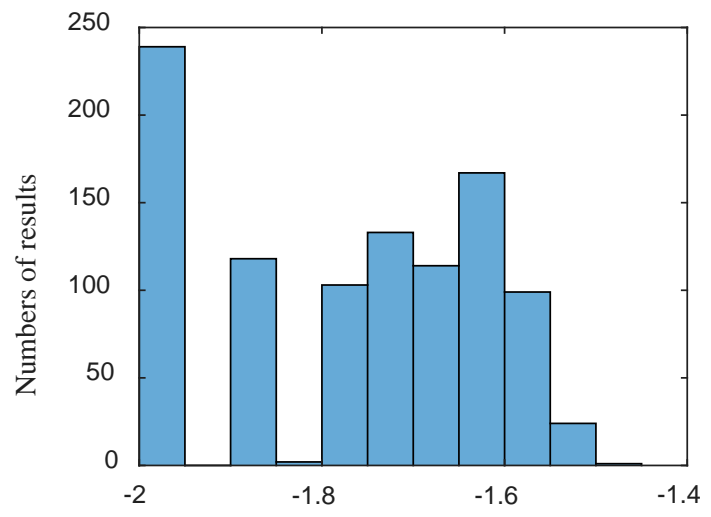

(a) Dsum

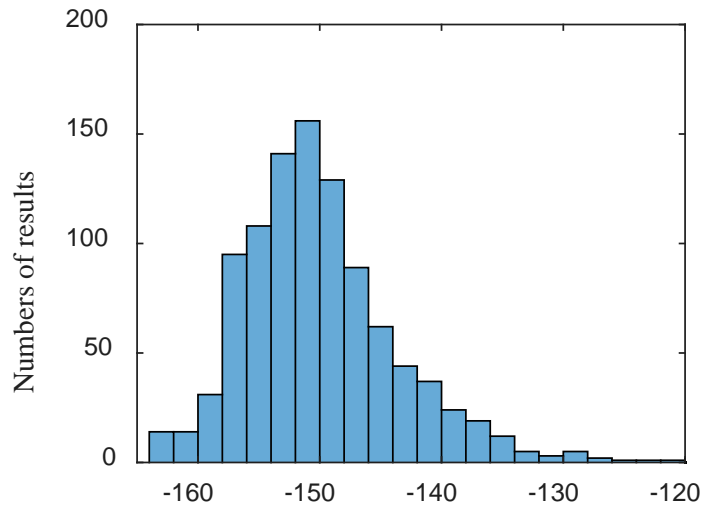

(b) Msum

Fig. 2 Distribution of Results

To get a clear view of performance, Fig. 2 shows the distribution of $M_{\text {sum }}$ and $D_{\text {sum. }}$. Here 1000 results of $9 \times 9$ package with 25 P/G pins are found by GA and each of them consumes around 3 seconds. Only 8 optimal packages (result in Fig. 3) with the smallest $f$ value is found and the $M_{\text {sum }}$ and $D_{\text {sum }}$ of the them are -163.92 and -2 respectively. 


\section{Construction of Pin Assignment}

A good return path quality requires a uniform $\mathrm{P} / \mathrm{G}$ pins distribution. For a 1-dimension package, to get a uniform $\mathrm{P} / \mathrm{G}$ pin assignment is easy. However, things become complex in a 2-dimension package. The construction procedure is as follows:

1) Initialize scalar $d_{\text {shift }}, d_{\text {inc }}, d_{\mathrm{t}}$ and a vector $\boldsymbol{p}_{1}$ with alternatively arranged power (P) pins, ground $(\mathrm{G})$ pins and signal $(\mathrm{S})$ pins.

2) Update vector $\boldsymbol{p}_{i+1}=\operatorname{shift}\left(\boldsymbol{p}_{i}\right)$.

3) Update package matrix $\boldsymbol{P}$ with $\boldsymbol{P}_{i \times \mathrm{d}_{\mathrm{t}} \text {-th column }}=\boldsymbol{p}_{i}$ and $\boldsymbol{P}_{(i+1) \times \mathrm{d}_{\mathrm{t}} \text {-th column }}=\boldsymbol{p}_{i+1}$.

4) Repeat step 2-3 until $(i+1) \times d_{\mathrm{t}}$ is larger than the number of columns in $\boldsymbol{P}$.

5) Increase $d_{\text {shift }}$ and repeat step 2-4 to get $\boldsymbol{P}_{1}, \boldsymbol{P}_{2}, \ldots, \quad \boldsymbol{P}_{N}$.

6) Calculate $M_{\text {sum }}$ and $D_{\text {sum }}$ for each package.

7) Plot curves of $M_{\text {sum }}$ and $D_{\text {sum }}$, select the best package as optimal solution.

In step 1, Parameter $d_{\text {inc }}$ is used to control the number of S pin between two P/G pins and $d_{\mathrm{t}}$ controls the interval between $\boldsymbol{p}_{i}$ and $\boldsymbol{p}_{i+1}$. They are initialized according to the ratio between P/G pins and the total number of pins in the package. For a given package, we have:

$$
r=\frac{N_{\mathrm{P} / \mathrm{G}}}{N_{\text {total }}} \leq \frac{1}{d_{\text {inc }} \times d_{\mathrm{t}}} \#(5)
$$

To find the $d_{\text {inc }}$ and $d_{\mathrm{t}}$ that make $r$ as close to the actual ratio as possible for initializing step. Grid search method can be used.

In step 2, the function $\operatorname{shift}(x)$ works like a shift resister and the shift distance depends on $d_{\text {shift }}$.

In step 3, suppose $I_{\mathrm{M}}$ and $I_{\mathrm{D}}$ is the period of $M_{\text {sum }}$ and $D_{\text {sum }}$ respectively. For any package, we have:

$$
\begin{gathered}
I_{\mathrm{M}}=2 d_{\text {inc } \#(6)} \\
I_{\mathrm{D}}=d_{\text {inc }} \#(7)
\end{gathered}
$$
Because $D_{\text {sum }}$ may remain a constant and $M_{\text {sum }} \underset{\bullet \text { Ground }}{\text { vary power }} \underset{\text { o Signal }}{\text { always. }} N$ is chosen according to $I_{\mathrm{M}}$.

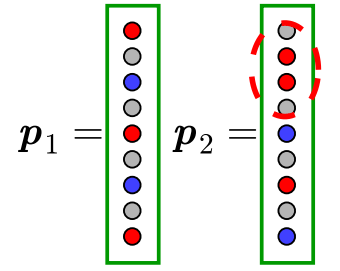

(a) Before Compensation

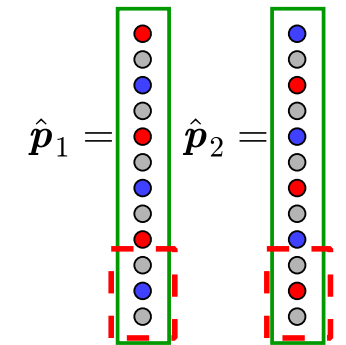

(b) After Compensation



(c) Constructing

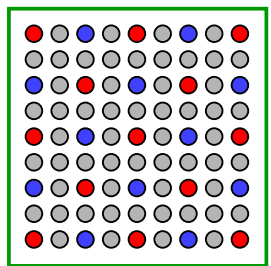

(d) Final Result

Fig. 3 Construction Procedure of a $9 * 9$ package

Fig. 3 shows the construction procedure of a $9 \times 9$ package with $25 \mathrm{P} / \mathrm{G}$ pins, here $d_{\text {shift }}=2$, $d_{\text {inc }}=1$ and $d_{\mathrm{t}}=1$. As show in Fig. 3 (a), the uniform scheme will be broken after shifting directly. To address this problem, some extra pins will be added on the tail of $\boldsymbol{p}$. The number of them can be calculated by

where $l$ is the length of $\boldsymbol{p}$.

$$
N_{\text {add }}=2 d_{\text {shift }}-\bmod \left(l, 2 d_{\text {shift }}\right) \#(8)
$$

Before shifting, $\boldsymbol{p}_{2}$ cannot maintain the uniform scheme, but $\hat{\boldsymbol{p}}_{2}$ can after compensating $\boldsymbol{p}_{2}$. The extra pins will be abandoned when $\widehat{\boldsymbol{p}}_{2}$ is used to construct $\boldsymbol{P}$.

Several pin assignment schemes will be constructed while changing the shifting distance $d_{\text {shift }}$. Fig. 4 shows the Numeric curves of packages. On this package, $D_{\text {sum }}$ remains a constant and $M_{\text {sum }}$ changes periodically. This plot shows the best shifting distance is 2. After finishing all steps, the result constructed by proposed method is the same as the best found by GA. 


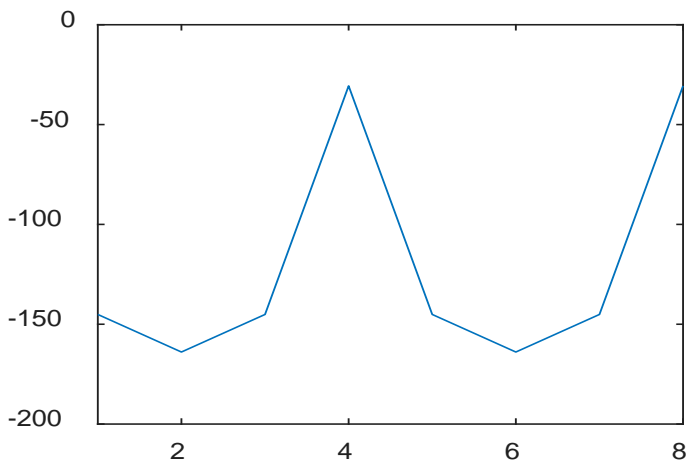

(a) Msum vs Distance

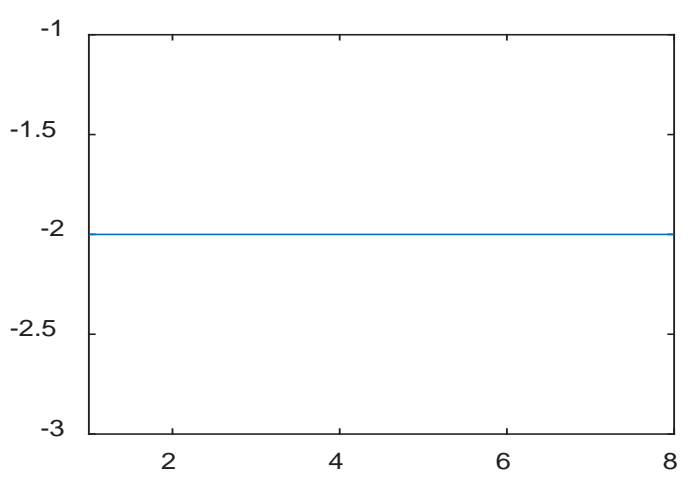

(b) Dsum vs Distance

Fig. 4 Numeric Curves of Results

\section{Experiment Results}

The Xilinx FPGA XC7VX485T is chosen to validate our construction method. The pinout of this device comprises of three parts: core power part (shown in the orange box), differential-pair part (shown in the red box) and GPIO part. Each part is constructed separately. Fig. 5 (a) shows the solution found by GA [4], it takes 30 minutes. In another work [6], it has been reduced to tens of second. Fig. 5 (b) shows another solution constructed by proposed method.

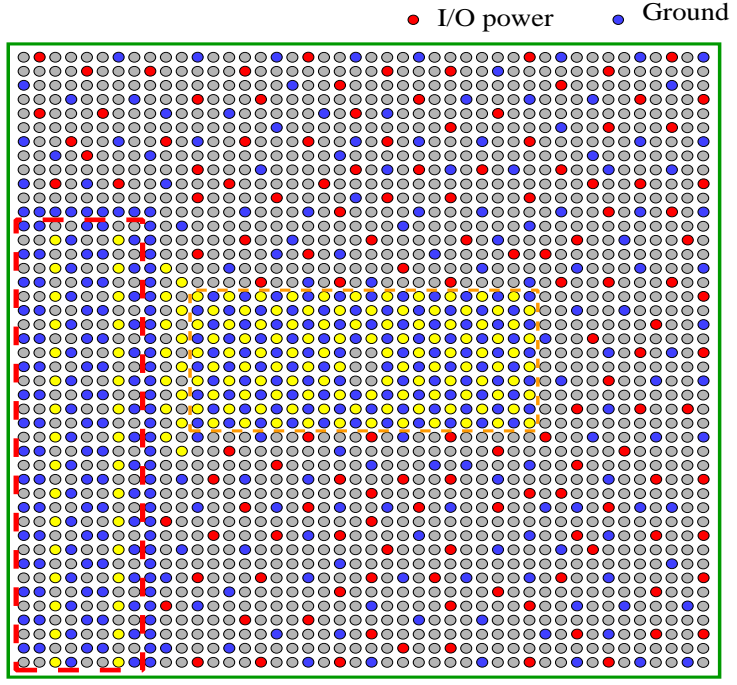

(a) GA

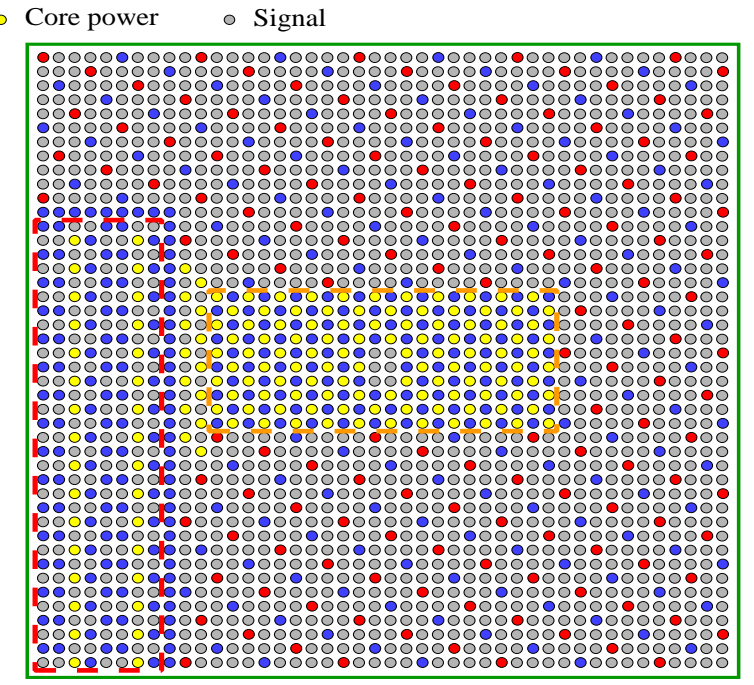

(b) Ours

Fig. 5 Experiment Results

The core power part and differential-pair part are the same since they are both small packages. By comparing the GPIO part of each solution. The pin assignment scheme of our solution is more uniform. To make the difference more obvious, a technique called color map assessment [6] is used.

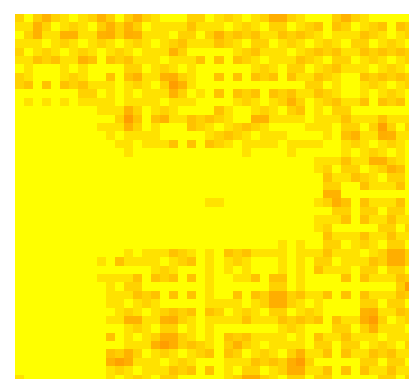

Fig. 6 Colormap of Solutions 
Fig. 6 shows the color map of the solutions. In Fig. 6 (a) and (b), light yellow area shows the good return path quality while dark area shows the worst. In Fig. 6 (c) and (d), the blue (cool) area show a negative inductance which means good signal integrity and that of the red(hot) area is poor.

\section{Conclusion}

In this paper, a fast pin assignment construction method for large-scale BGA package is proposed. It's guided by the prior knowledge of the optimal solution. It's highly efficient in package designing. If there is a need for a further optimal pin assignment scheme, the solutions found by the proposed method can act as the initial solutions in heuristic algorithms for further optimization [7].

\section{Acknowledgement}

In this paper, the research was sponsored by the National Natural Science Foundation of China (Project No.61473322). Nature Science Foundation of Guangdong (Project No.2016A030310335) and SYSU-CMU Shunde International Joint Research Institute Major Research Project (Project No.20170106).

\section{References}

[1] 2013 ORTC Technology Trend Targets. International Technology Roadmap for Semiconductors (ITRS) Anline]. Available: https://www.dropbox.com/sh/qz9gg6uu4kl04vj/AADD7ykFdJ2ZpCR1LAB2XEjIa?dl=0\&pre view=2013ORTC_DetailedTable.pdf

[2] An approach to topological pin assignment [J]. IEEE Transactions on Computer-Aided Design of Integrated Circuits and Systems, 19843 (3) 250-255.

[3] Zhang Lihong, Q. J. Zhang. Optimal pin-assignment for ground noise minimization in IC packages and connectors. 1996 Proceedings 46th Electronic Components and Technology Conference [C]. Orlando: IEEE, 1996. 761-764

[4] Pin assignment optimization for large-scale high-pin-count BGA packages using genetic algorithm [J]. IEEE Transactions on Components, Packaging and Manufacturing Technology, 20155 (2) 232-244.

[5] Pin Assignment Optimization for Large-Scale High-Pin-Count BGA Packages Using Simulated Annealing [J]. IEEE Transactions on Components, Packaging and Manufacturing Technology, 20166 (10) 1465-1474.

[6] Static template for fast power/ground pin assignment of large-scale BGA packages using genetic algorithm [J]. IEEE Transactions on Components, Packaging and Manufacturing Technology, 20155 (8) 1142-1151.

[7] Pedro A. Diaz-Gomez, Dean F. Hougen. Initial Population for Genetic Algorithms: A Metric Approach. International Conference on Genetic and Evolutionary Methods, GEM 2007 [C]. Las Vegas: CSREA Press, 2007. 43-49 